\title{
REVIEW
}

\section{Taming the Trojan horse: optimizing dynamic carrier cell/oncolytic virus systems for cancer biotherapy}

\author{
AT Power and JC Bell \\ Centre for Cancer Therapeutics, Ottawa Health Research Institute, University of Ottawa, Ottawa, Ontario, Canada
}

\begin{abstract}
Live cells offer unique advantages as vehicles for systemic oncolytic virus (OV) delivery. Recent studies from our laboratory and others have shown that virus-infected cells can serve as Trojan horse vehicles to evade antiviral mechanisms encountered in the bloodstream, prevent uptake by off-target tissues and act as microscale factories to produce OV upon arrival in tumor beds. However to be employed effectively, OV-infected cells are best viewed as dynamic biological systems rather than static therapeutic agents. The time-dependent processes of infection and in vivo cell trafficking will inevitably vary depending on which particular OV is being delivered, as well as the type of carrier cells (CC) employed. Understanding these parameters with respect to each unique $\mathrm{CC} / \mathrm{OV}$ combination will therefore be required in order to effectively evaluate and harness their potential in preclinical study. In the following review, we
\end{abstract}

discuss how early studies of OV delivery led us to investigate the use of cell carriers in our laboratory, and the approaches we are currently undertaking to compare the dynamics of different CC/OV systems. On the basis of these studies and others it is apparent that the success of any cell-based system for OV delivery rests upon the coordinated timing of three sequential phases-(1) ex vivo loading, (2) stealth delivery and (3) virus production at the tumor site. While at the current time, the timing of these processes are coupled to the natural cycle of infection and in vivo trafficking properties innate to each cell virus system, a quantitative delineation of their dynamics will lay the foundation for engineering $\mathrm{CC} / \mathrm{OV}$ biotherapeutic systems that can be clinically deployed in a highly directed and controlled manner.

Gene Therapy (2008) 15, 772-779; doi:10.1038/gt.2008.40; published online 27 March 2008

Keywords: vesicular stomatitis virus; oncolytic virus; cancer therapeutics; immunity; cell-based delivery; biotherapeutics

\section{Barriers to oncolytic virus delivery: lessons from the study of oncolytic vesicular stomatitis virus}

A number of different oncolytic virotherapeutics have been developed to date; preclinical data regarding their unique mechanisms of tumor-specific replication, killing and potential to induce antitumor immunity have been reviewed previously, ${ }^{1}$ as has the recent experience with some of these agents in clinical trials. ${ }^{2}$ Work in our laboratory initially uncovered that innate immunesignaling pathways are defective in many types of cancer cells, rendering them particularly susceptible to infection and cytolysis by vesicular stomatitis virus (VSV). ${ }^{3}$ These findings prompted us to further investigate the oncolytic capacity of attenuated VSV strains in immunocompetent murine models. ${ }^{4}$ Although lasting disease remission could often be achieved following systemic administration, it was also apparent from these studies that there are unique challenges to achieving VSV delivery in vivo.

Correspondence: Dr JC Bell, Centre for Cancer Therapeutics, Ottawa Health Research Institute, University of Ottawa, 503 Smyth Road, Ottawa, Ontario, Canada K1H 8L6.

E-mail: jbell@ohri.ca

Received 14 February 2008; accepted 16 February 2008; published online 27 March 2008
Innate barriers to systemic OV delivery

The circulatory system is an unwelcoming environment for any virus. However, it remains the best avenue for delivering therapeutics to undetectable or physically inaccessible sites of tumor metastasis. Understanding the impact of innate and acquired immune barriers to systemic OV delivery has therefore been an area of intense research in recent years. Our laboratory has focused on modeling the OV-host interactions that influence intravenous (i.v.) delivery of oncolytic VSV (AV1) in immunocompetent animals. ${ }^{4-6}$

Early investigations in this system revealed that successful delivery to both lung and hind flank tumors could be achieved when acceptable therapeutic doses (at least 100-fold below maximum tolerated dose) were i.v. administered to naive animals. ${ }^{4}$ Viral titers, fluorescent transgene expression and immunohistochemical analysis independently confirmed successful delivery and amplification of virus within tumors. ${ }^{6}$ However, subsequent biodistribution experiments revealed that i.v. delivery was far from efficient $-<0.001 \%$ of the administered virions were found to reach hind flank tumors, as the vast majority of the input dose was immediately taken up from the circulation by the liver and spleen. ${ }^{6}$ These organs also act as innate barriers to delivery in other OV systems, an effect generally attributable to phagocytic uptake by resident macrophages of the reticuloendothelial system. ${ }^{7}$ 
These studies revealed that although i.v. delivery of VSV was less than perfect even in naive animals, sufficient infectious virus could reach the tumor to initiate a robust infection and mediate a significant therapeutic response. ${ }^{4}$ Thus innate barriers, while highly effective at clearing virus from the bloodstream, can ultimately be overwhelmed at VSV doses well within the practical limits of safety and manufacturing capacity.

\section{Adaptive barriers to systemic OV delivery}

Unlike conventional therapeutics, the pharmacokinetic parameters of $\mathrm{OV}$ delivery change over time as treated animals mount an adaptive immune response. Thus, having established that VSV could be delivered to tumors in naive animals, we were next interested in examining how adaptive defenses might affect repeat dosing regimens. In this respect, the murine balb/c model has provided a useful model, as VSV infection induces a rapid and robust B- and $\mathrm{T}$-cell response, leading to the formation of high-titer antiviral antibodies that play a crucial role in virus clearance. ${ }^{8-10}$

We found that the recombinant oncolytic strain of VSV developed in our laboratory (AV1) induced serum neutralizing antibodies with kinetics indistinguishable from the previously reported responses to wild-type strains. ${ }^{5}$ VSV-neutralizing antibodies were detectable at least as early as 4 days following treatment with a single virus dose and reached peak levels (1/3200-1/6400) within 2-3 weeks, remaining at these elevated levels for at least several months. Administration of repeated doses served to further boost antibody levels, leading to a two- to fourfold increase in plateau titers. Consequently, we found that the evolution of this antibody response is a serious impediment to VSV delivery. In contrast to the case of naive animals, VSV delivery was completely ablated in mice that had received a previous dose of the virus up to several months in advance, as we reproducibly found a lack of detectable virus transgene expression or viral titers in tumors. ${ }^{5}$ Passive transfer experiments revealed that this adaptive state of sterilizing immunity to VSV treatment was mediated by serum antibodies, whereas adoptively transferred T cells had no impact. ${ }^{5}$ Immune serum containing anti-VSV antibody was similarly potent in vitro, able to neutralize an entire therapeutic dose (approximately 10e8 PFUs (plaque-forming units)) at a 1/10 dilution (AT Power and JC Bell, unpublished data). Unlike the innate barriers discussed in the preceding section, it was not possible to saturate and overwhelm this induced antibody at sublethal virus doses in vivo (AT Power and JC Bell, unpublished data).

Thus antiviral antibodies are induced during treatment and intercept therapeutic virus even before it reaches the tumor. Long-lived immunological memory is activated, leading to elevated antibody titers capable of mitigating i.v. delivery for months, if not years after the initiation of treatment. These findings suggested that a carrier system capable of concealing viral antigen en route to the tumor site might be the best way to achieve consistent delivery of repeated OV doses in the face of evolving adaptive immunity.

\section{Carrier cells: Trojan horse vehicles for systemic OV delivery}

Early in vivo experimentation with OVs had suggested that infected virus-producing cells could also mediate antitumor effects when administered in the place of naked virions. ${ }^{3,11}$ This hinted that cellular carriers might be used as Trojan horse vehicles to shield OV from neutralization following systemic administration, and act as in situ virus factories once arriving at the tumor site. Having established a reliable murine system to model OV therapy in the context of evolving host immunity, 4,5 we were able to put this concept to the test. We hypothesized that carrier cells (CCs), infected ex vivo and then infused i.v., would enable escape from antiviral defenses if they could reach the tumor site during eclipse phase (prior to viral protein synthesis and virion release) and subsequently release progeny virions to infect surrounding cancer cells. Early studies indicated that this was indeed the case, as we saw that infected cells delivered virus to murine lung tumors to establish a robust infection of the malignant tissue. ${ }^{5}$ More importantly, cellular delivery and subsequent tumor infection was unaffected by the presence of circulating antiviral antibody at high titers that entirely neutralized delivery of naked virions. ${ }^{5}$ These experiments provided exciting proof-of-concept that infected cells could indeed act as Trojan horse vehicles to smuggle an OV, such as VSV, past circulating antibody molecules into tumor beds.

Similar studies by other groups have shown that cell carriers can also deliver oncolytic measles virus to tumors in the presence of virus-neutralizing antibody. ${ }^{12,13}$ However, Ong et al. ${ }^{12}$ observed that this effect was antibody dose-dependent in their system, as tumor infection remained unattainable at higher concentrations of human neutralizing antibody even when carriers were used. Failure to infect tumors at high antibody concentrations could have been a result of neutralization of carriers cells prematurely expressing viral surface proteins within the bloodstream, as the kinetics of measles antigen expression were not considered in this study. It could also be a result of the general inefficiency of delivery with the particular cell type examined, as the primary T-cell carriers did not release progeny virus, but rather relied on direct cell contact to transfer infectivity by fusion with tumor cells. Additionally, only $1.5 \%$ of virus-loaded $\mathrm{T}$ cells accumulated in the target tumor tissue in the model used for these experiments.

In contrast, more efficient CC delivery may help to overcome antiviral immunity. In the VSV system, we have observed robust antibody escape using highly virus-permissive carcinoma cell carriers, ${ }^{5}$ which are capable of producing upward of 100 progeny virions per cell (AT Power and JC Bell, unpublished data). Furthermore, these types of solid tumor-derived carriers accumulated primarily at the site of the target tumors (in the lungs) following i.v. administration in these experiments. ${ }^{5}$

Comparing these studies highlights the fact that successful cell-based delivery of OV is highly dependent on the kinetics of virus replication, the in vivo trafficking properties of the CCs and the quantity of virions the cells can produce. Numerous different $\mathrm{CC} / \mathrm{OV}$ combinations are currently under investigation ${ }^{5,11-19}$ and in essence, 
each represents a unique biotherapeutic system for which these time-dependent parameters must be considered to achieve optimal tumor delivery. In the remainder of this article, we discuss the main conditions that must in theory be met to achieve true Trojan horse OV delivery, and relate some of the lessons learned from our ongoing efforts to evaluate the properties of various established cell lines as VSV carriers.

\section{Timing is of the essence: three critical phases for successful cell-based delivery of the OV payload}

Ultimately the success or failure of any cellular OV delivery system rests on the proper coordination of three critical phases in both space and time, as illustrated in Figure 1. First, loading of the CCs is carried out ex vivo, second, delivery of cells to the tumor site is achieved via the circulation and third, release of virus must occur within the tumor bed. Once initiated, the timing of this sequence is inextricably linked to that of the OV life cycle within the particular cell type used for delivery. A detailed understanding the dynamics of these processes is therefore required to ensure that CCs reach the right place at the right time.

\section{Phase l: ex vivo loading}

Essentially the goal of the ex vivo loading phase is to productively infect as many $\mathrm{CCs}$ as possible in the shortest possible time. The reasoning for this is fairly straightforward; the more CCs infected, the greater the proportion of injected cells that will be capable of bringing virus to the tumor, and in turn the higher the potential oncolytic dose. Therefore cells must be exposed to virus for sufficient time for uptake to occur but no longer, as increasing the amount of time that passes before injection increases the likelihood that viral antigen expressed on the cell surface will be exposed to host antibodies during the stealth delivery phase (Figure 1; also see phase II below).

For each $\mathrm{CC} / \mathrm{OV}$ system the efficiency of a given loading protocol is readily assessed by counting the proportion of cells that express viral protein during the first infection cycle following uptake. Flow cytometric analysis of viral transgene expression provides a useful tool for such analysis. In our studies, infection of adherent CC lines with VSV at an MOI (multiplicity of infection) of 10 PFUs per cell led to nearly $100 \%$ loading after a period of $1 \mathrm{~h}$ (AT Power and JC Bell, unpublished data). However, loading of non-adherent cell lines, necessary to study leukocyte carrier systems, appears to be less efficient. For example, suspension of Jurkat cell lines in 10 PFUs per cell of VSV ( $10^{6}$ cells per $\left.\mathrm{ml}\right)$ for the

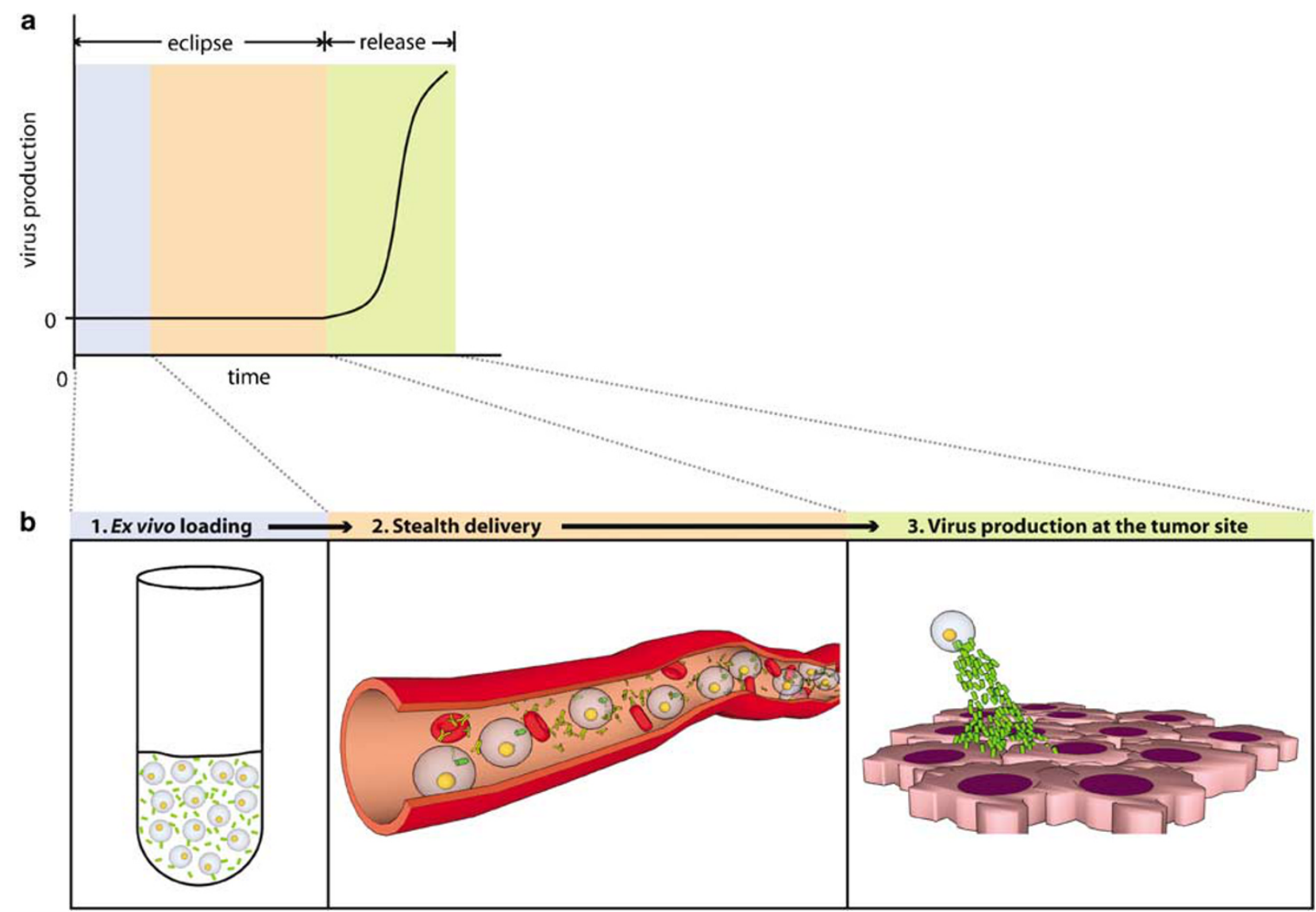

Figure 1 Three sequential phases are critical for carrier cell (CC)-based delivery of replicating oncolytic viruses (OV). (a) Typical kinetics of OV growth within permissive CCs. Following addition of virus at $t=0$, cellular carriers undergo an eclipse period prior to the onset of viral protein synthesis, exponential amplification and release of progeny virions. (b) Three sequential phases of CC/OV delivery, mapped to their ideal timing within the viral growth cycle shown as in a). 
same $1 \mathrm{~h}$ leads to uptake and infection of only $67 \%$ of the total cell population (Figure 2). Lengthening the loading phase or enhancing uptake by other means, such as centrifugation, may therefore be necessary to maximize loading of non-adherent leukocytic CCs. In addition to variation between $\mathrm{CC}$ types, optimal uptake conditions are likely to vary between OVs; for example, standard infection protocols for Vaccinia virus infection typically specify $2 \mathrm{~h}$ rather than the $1 \mathrm{~h}$ routinely used for VSV.

While the optimizing the efficiency of virus uptake may seem of trivial importance with respect to therapy in vivo, it is in fact a crucial consideration for achieving effective immune evasion and tumor delivery. Once they are injected into the circulation, premature expression of viral protein on the surface of CCs is likely to betray the presence of the $\mathrm{OV}$ payload hidden within, allowing antiviral antibodies to bind and target the Trojan horse vehicle for destruction by complement proteins, phagocytes or cytotoxic cells. The length of this eclipse or latent period is therefore an important parameter to consider for each different $\mathrm{CC} / \mathrm{OV}$ system. In our initial immune evasion studies using carcinoma carriers to deliver VSV, ex vivo loading followed by western blotting analysis revealed minimal viral protein synthesis within the first $3 \mathrm{~h}$ of infection. Therefore in subsequent experiments this data were used to guide the timing of loading, harvesting and administration of CCs to animals, and we found that cells injected into mice $2-3 \mathrm{~h}$ following infection could successfully avoid neutralization by antiviral antibodies. ${ }^{5}$ As various other $\mathrm{CC} / \mathrm{OV}$ systems are explored, therapeutically relevant measurement of antigen exposure kinetics could also be achieved by analyzing antiviral antibody binding to the CC surface by flow cytometry. In summary, the timing of $\mathrm{OV}$ protein expression in infected CCs specifies the maximum allowable time for ex vivo loading. Determination of this crucial parameter therefore allows optimal CC loading and therapeutic administration to be achieved within a time window that will not compromise the stealth of these Trojan horse vehicles.

\section{Phase II: stealth delivery}

This phase involves stealthy passage of OV-laden cells through the circulation and their arrival within tumor deposits (Figure 1). As for phase I, the timing of the oncolytic viral life cycle is a crucial determinant of the success of this phase. To achieve true, 'direct-to-tumor' virus delivery requires that CCs accumulate in tumor beds before progeny virions are released. Furthermore, to act as Trojan horse vehicles and successfully evade antiviral antibody, CCs should ideally reach the tumor site even before viral antigen is displayed on their surface. To ensure that infected CCs arrive at their tumor destination on time, it is necessary to understand the kinetics of their dissemination following systemic administration.

For example in the case of VSV, permissive cell types produce substantial amounts of viral protein by $6 \mathrm{~h}$ or less postinfection, so CCs will have ideally arrived at their destination before this time. We have relied on bioluminescent imaging (BLI) to track the biodistribution of CCs in real time following i.v. administration to mice. Using this approach we initially transduced several solid tumor cell lines with a lentivirus encoding Renilla luciferase and found that they exhibited similar biodistribution patterns-rapid accumulation and arrest in lung tissue within $30 \mathrm{~min}$ following tail-vein injection, where they remained for at least another $24-48$ h. ${ }^{5}$ In the same experiments, tumor-specific expression of a viral
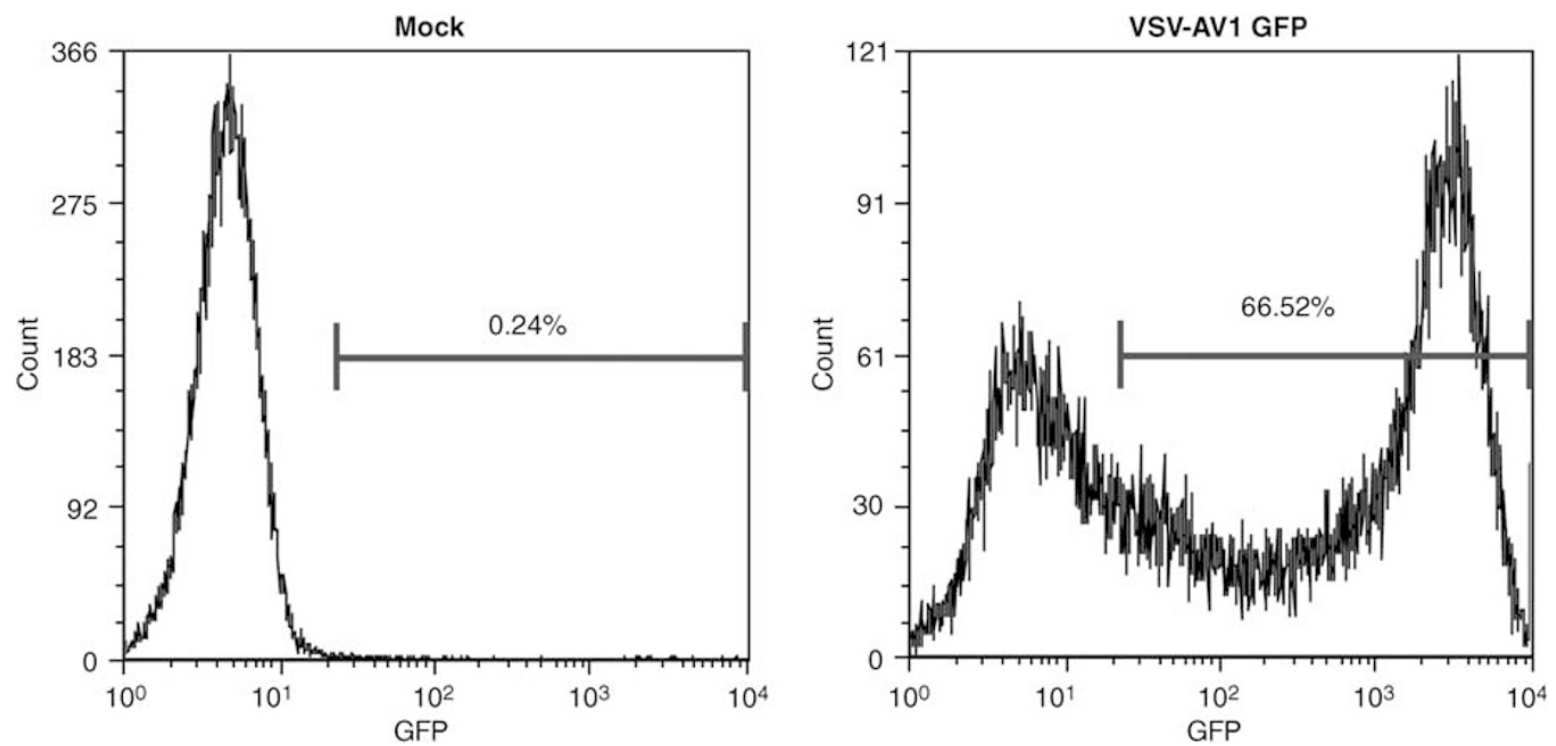

Figure 2 Assessing ex vivo loading of leukocytic carrier cells (CC). Jurkat human T cells were suspended in 10 PFUs (plaque-forming units) per cell vesicular stomatitis virus (VSV)-AV1-green fluorescent protein (GFP) for $1 \mathrm{~h}$ at a concentration of $10^{6}$ cells per ml. Cells were then pelleted, washed twice with $10 \mathrm{ml}$ phosphate-buffered saline, resuspended in $10 \mathrm{ml}$ Dulbecco's modified Eagle's medium (DMEM)+10\% fetal calf serum and incubated at $37^{\circ} \mathrm{C}$ for an additional $7 \mathrm{~h}$. Mock or VSV-AV1-GFP-infected cells were analyzed by flow cytometry and percentages of GFP-positive cells are shown on each histogram. 
firefly luciferase transgene persisted long after clearance of the carriers, confirming successful release and delivery of $\mathrm{OV}$ at the tumor site..$^{5}$ Therefore the biodistribution profiles of solid tumor cell carriers indicated that they were effective for delivery to tumors growing in the lungs or other directly injectable sites, but it seemed apparent that delivery of $\mathrm{OV}$ to more disseminated sites would require carriers capable of escaping arrest in narrow capillary beds. This is true of the L1210 murine lymphocytic leukemia cell line, which we have seen bypasses the lungs and rather accumulates in the spleen within several hours after i.v. injection (Figure 3a; Power et $a l .{ }^{5}$ ). In contrast, the human myeloid leukemia K562, another cell line whose distribution we have examined a

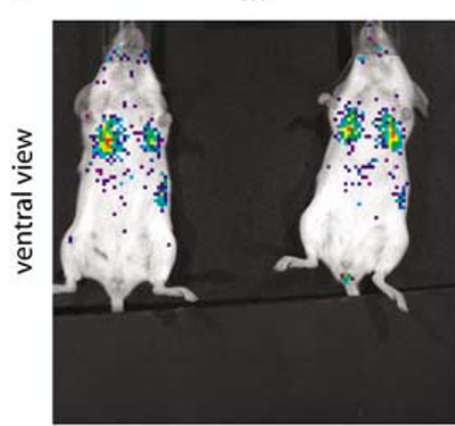

$1 \mathrm{~h}$

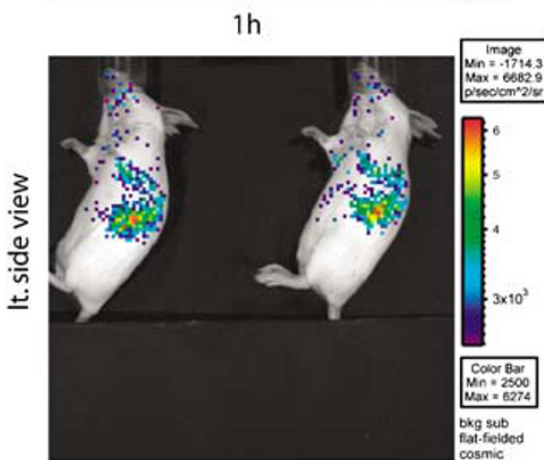

b
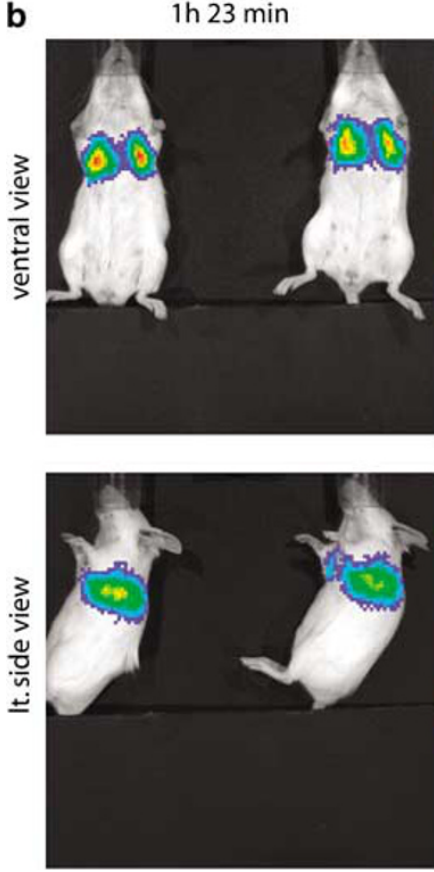
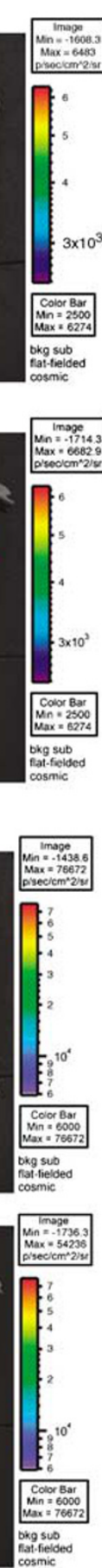

7h $50 \mathrm{~min}$

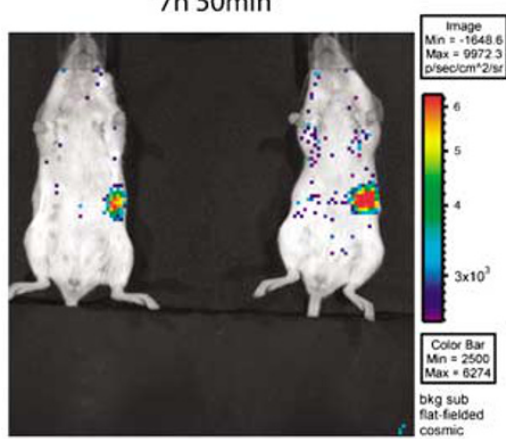

$7 \mathrm{~h} 50 \mathrm{~min}$

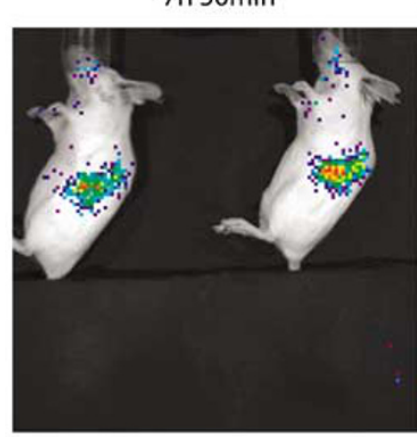

$8 \mathrm{~h} 11 \mathrm{~min}$
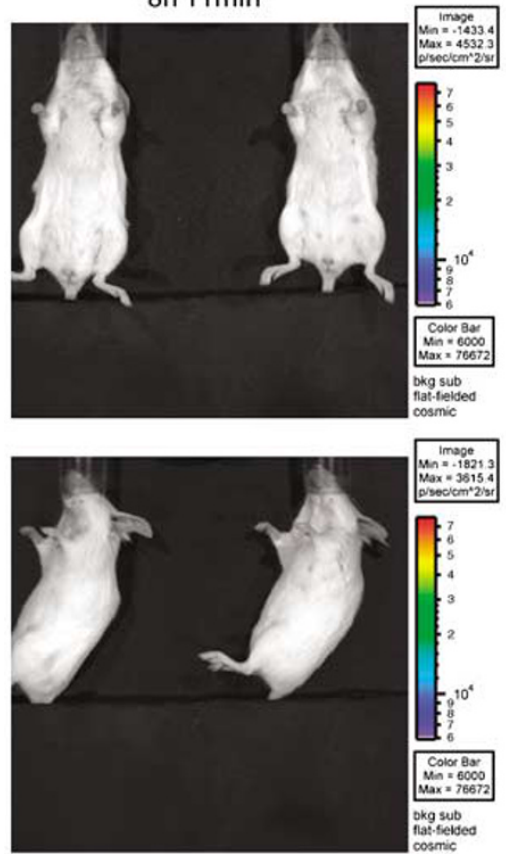

$26 \mathrm{~h}$

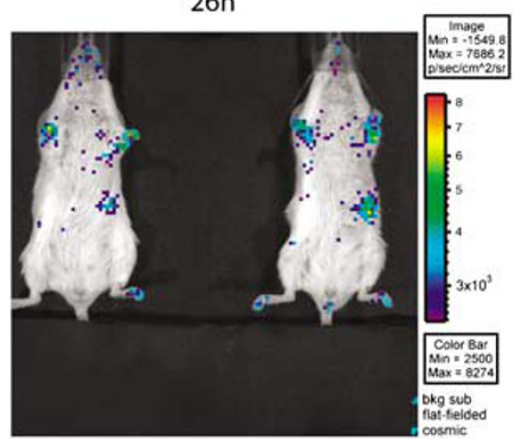

$26 \mathrm{~h}$

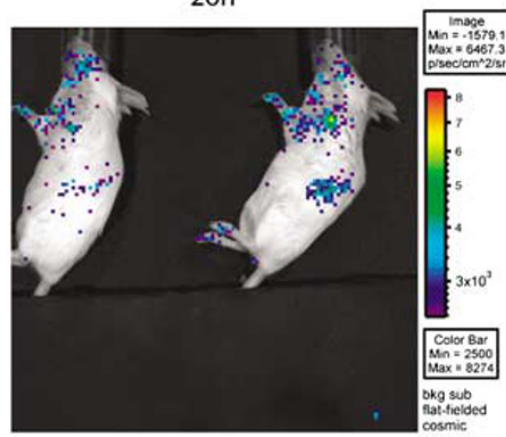

$26 \mathrm{~h} 23 \mathrm{~m}$
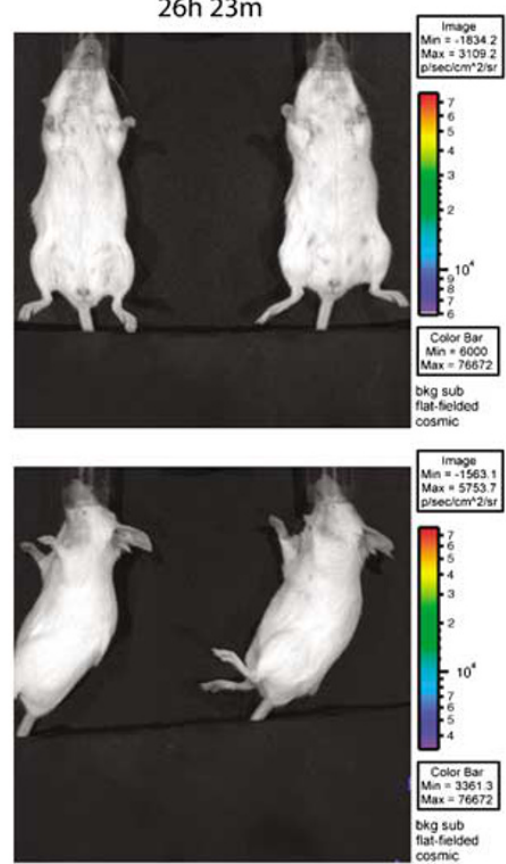

Figure 3 Assessing the trafficking dynamics of leukocytic carrier cells (CC) in vivo. L1210 murine lymphocytic leukemia cells (a) or K562 human myeloid leukemia cells (b) stably transduced with the firefly luciferase gene were administered to mice by tail-vein injection $\left(10^{6}\right.$ cells per mouse). To follow the fate of injected cells, bioluminescent images were acquired at time points indicated, as described previously. ${ }^{5}$ Ventral and left side views of two mice that were treated and imaged together at each time point are shown. 
by BLI, accumulated, like solid tumor lines, exclusively within lung tissue (Figure $3 b$ ). Therefore the ability to circulate through blood vessels may be a property of some, but not all types of leukocytic carriers.

In general, these examples demonstrate one important lesson learned from experiments to date-different CC lineages can exhibit quite distinct patterns of dissemination when injected into blood vessels. We are continuing to investigate these patterns using a large panel of different murine and human leukemia cell types to learn which variables-such as cell size or cell surface adhesion receptor expression profiles-determine innate CC tropisms. It would also be desirable to engineer CCs targeted specifically to the tumor microenvironment, which could be achieved by introducing cell surface proteins that bind receptors on neovasculture or the tumor cell surface such as those that have been designed to retarget the tropism of virotherapeutics previously. ${ }^{20-26}$

Another issue that has been illuminated by our BLI studies is the clearance kinetics of histoincompatible CC lines. Although both allogeneic and xenogeneic (human) cell lines appear equally capable of delivering VSV to lung tumors in mice, bioluminescent signal from allogeneic lines persisted for up to $24-48 \mathrm{~h}$ (Figure 3a; Power et $a l .5$ ), whereas xenogeneic carriers seem to be cleared more rapidly, within $<8 \mathrm{~h}$ of administration to mice (Figure 3b; Power et al..$^{5}$ ). These findings are consistent with known mechanisms of immunological rejection-activation of adaptive $\mathrm{T}$-cell responses are required for allograft rejection, whereas xenografts can be targeted much more rapidly by innate circulating factors such as natural antibodies and complement proteins. $^{27}$ Although this clearance did not limit VSV delivery to tumors in the lung model we have examined, as OV release occurred before CCs were eliminated, if loaded with a slower-replicating OV virus or used to target tumor sites that take longer to access, rapidly cleared xenogenic cells might not have the opportunity to deliver their cargo to the tumor site. It also remains to be seen whether allo/xenogeneic carriers induce an adaptive response that might inhibit delivery with repeated dosing.

Although there is certainly more to learn, BLI has already provided valuable insight into the dynamics of CC delivery in vivo. Noninvasive molecular imaging technology should continue to be extremely useful as we seek to manipulate CC tropism to promote timely infiltration of tumor beds at diverse anatomical locations.

\section{Phase III: virus production at the tumor site}

Upon reaching the tumor bed, the role of each CC shifts from delivery vehicle to in situ OV factory (Figure 1). Depending on the $\mathrm{CC} / \mathrm{OV}$ combination, each OVinfected cell may release a localized burst of up to hundreds of viral particles (as in production of VSV by permissive carrier lines). This unique ability offers the potential for significant dose amplification following delivery to the target tissue if cell types capable of high viral productivity are employed.
As for other parameters, the total quantity and kinetics of virus release is a unique property of each $\mathrm{CC} / \mathrm{OV}$ combination. To evaluate candidate CC types, this variable is readily measured by loading the cells and following virus production in vitro. For the sake of comparing virus production between candidate carrier lines, it is straightforward to measure the average number of virions released per cell over time to construct classic one-step growth curves. Applying this analysis to some of the CC lines used to deliver oncolytic VSV in our laboratory, we have measured an average productivity on the order of 100-200 PFUs per cell following infection of permissive carcinoma cell lines of either human or murine origin (AT Power and JC Bell, unpublished data). In contrast, permissive leukemia cell lines grown in suspension culture have shown a trend toward lower productivity, on the order of 10-90 PFUs per cell, when infected at comparable MOIs (AT Power and JC Bell, unpublished data). However as discussed under the topic of cell loading, the efficiency of viral uptake also appears to be much diminished when cells are infected in suspension, since even at high MOIs $(\geqslant 10$ PFUs per cell) we have seen that ex vivo uptake is often submaximal (Figure 2; AT Power and JC Bell, unpublished data). Thus to accurately measure the average productivity of each candidate CC line, it is important to also determine the efficiency of uptake under the relevant infection conditions, which should ideally be as close a possible to $100 \%$.

Despite the limitations of these studies to date, they also highlight differences between normal and transformed carriers. As discussed above, the activated primary $\mathrm{T}$ cells used as carriers as described by Ong et al. ${ }^{12}$ produced no detectable measles virus progeny, despite showing synthesis of the green fluorescent protein (GFP) viral transgene. In a direct comparison, Iankov and co-workers also reported decreased oncolytic measles virus productivity in normal cell types compared to transformed cell lines. ${ }^{13}$ So although autologous primary cell carriers offer attractive benefits in terms of safety and immunological compatibility, special pharmacological or genetic manipulation may be necessary before they can be effectively used to produce OVs that have been engineered to replicate preferentially in transformed cells. In contrast, transformed cell lines generally produce large quantities of $\mathrm{OV}$ and are therefore immediately available as robust carriers for preclinical and clinical studies.

A final issue that may relevant to future studies of CC populations is that of cell to cell variation in viral production capacity. Heterogeneous gene expression profiles within $\mathrm{CC}$ populations, for example due to variation in differentiation or cell cycle stage, could lead to differences in the quantity of progeny virions produced by each cell. It remains unknown whether such heterogeneity is characteristic of the types of CCs currently under study, as it is a rather cumbersome task to quantitate viral production by single cells of infected populations. Nonetheless such analysis is possible using basic virological techniques, and has indeed been used in the past to illuminate subpopulations of cultured cells with marked differences in viral productivity. ${ }^{28}$ Cell to cell differences in viral productivity would have an impact on their utility as carriers in vivo, as each is likely to behave as an independent virus factory following 
delivery to a particular region of the tumor. Depending on the source of any such heterogeneity, measures such as cell cycle synchronization or fluorescence-activated cell sorting might be used to ensure that each CC administered is capable of maximal virus production upon arrival at the tumor.

\section{Prospects for the future: toward programming dynamic CC/OV systems}

The above has outlined some of the important points to consider in evaluating and comparing the potential of different cell types as OV carriers. Currently, efforts in our laboratory are focused on applying this framework to compare a large panel of established cell lines of solid tissue and hematological origin. Taken together with the results of other studies (reviewed in Power and Bell ${ }^{19}$ ) that have examined cytokine-induced killer cells, ${ }^{14}$ primary leukocytes, ${ }^{12,13}$ mesenchymal stem cells ${ }^{15,16}$ and tumor antigen-specific T cells, ${ }^{29}$ some of the key issues associated with the cell-based delivery approach are already beginning to become apparent. With respect to tumor targeting, for example, it is clear that many cell types are severely hampered by their inability to pass through capillary beds, causing accumulation within nontumor-bearing tissues. ${ }^{5,16}$ Several studies however have indicated that leukocytic carriers are better able to circulate, 5,12,14 and therefore these cell types would seem the best platform for further efforts to refine the specificity of tumor targeting.

Ongoing study in this vein will allow the beneficial properties of diverse natural cell types to be exploited as systemic OV carriers, and will also delineate areas for further improvement. As our expertise in engineering biological systems grows, this knowledge could be used to move us closer toward reprogramming the natural trafficking and virus production dynamics of $\mathrm{CC} / \mathrm{OV}$ systems so as to ensure efficient execution of each sequential stage of delivery (Figure 1). This avenue of research should therefore continue to be a fruitful one, as the CC approach opens up many new angles from which to attack the ultimate challenge of achieving controlled, reliable delivery of OVs to metastatic tumor deposits in humans.

\section{References}

1 Parato KA, Senger D, Forsyth PA, Bell JC. Recent progress in the battle between oncolytic viruses and tumours. Nat Rev Cancer 2005; 5: 965-976.

2 Liu TC, Galanis E, Kirn D. Clinical trial results with oncolytic virotherapy: a century of promise, a decade of progress. Nat Clin Pract Oncol 2007; 4: 101-117.

3 Stojdl DF, Lichty B, Knowles S, Marius R, Atkins H, Sonenberg N et al. Exploiting tumor-specific defects in the interferon pathway with a previously unknown oncolytic virus. Nat Med 2000; 6: 821-825.

4 Stojdl DF, Lichty BD, tenOever BR, Paterson JM, Power AT, Knowles $\mathrm{S}$ et al. VSV strains with defects in their ability to shutdown innate immunity are potent systemic anti-cancer agents. Cancer Cell 2003; 4: 263-275.

5 Power AT, Wang J, Falls TJ, Paterson JM, Parato KA, Lichty BD et al. Carrier cell-based delivery of an oncolytic virus circumvents antiviral immunity. Mol Ther 2007; 15: 123-130.
6 Breitbach CJ, Paterson JM, Lemay CG, Falls TJ, McGuire A, Parato KA et al. Targeted inflammation during oncolytic virus therapy severely compromises tumor blood flow. Mol Ther 2007; 15: 1686-1693.

7 Fisher K. Striking out at disseminated metastases: the systemic delivery of oncolytic viruses. Curr Opin Mol Ther 2006; 8: 301-313.

8 Gobet R, Cerny A, Ruedi E, Hengartner H, Zinkernagel RM. The role of antibodies in natural and acquired resistance of mice to vesicular stomatitis virus. Exp Cell Biol 1988; 56: 175-180.

9 Leist TP, Cobbold SP, Waldmann H, Aguet M, Zinkernagel RM. Functional analysis of $\mathrm{T}$ lymphocyte subsets in antiviral host defense. J Immunol 1987; 138: 2278-2281.

10 Zinkernagel RM, Adler B, Holland JJ. Cell-mediated immunity to vesicular stomatitis virus infections in mice. Exp Cell Biol 1978; 46: 53-70.

11 Coukos G, Makrigiannakis A, Kang EH, Caparelli D, Benjamin I, Kaiser LR et al. Use of carrier cells to deliver a replicationselective herpes simplex virus-1 mutant for the intraperitoneal therapy of epithelial ovarian cancer. Clin Cancer Res 1999; 5: 1523-1537.

12 Ong HT, Hasegawa K, Dietz AB, Russell SJ, Peng KW. Evaluation of $\mathrm{T}$ cells as carriers for systemic measles virotherapy in the presence of antiviral antibodies. Gene Therapy 2007; 14: 324-333.

13 Iankov ID, Blechacz B, Liu C, Schmeckpeper JD, Tarara JE, Federspiel MJ et al. Infected cell carriers: a new strategy for systemic delivery of oncolytic measles viruses in cancer virotherapy. Mol Ther 2007; 15: 114-122.

14 Thorne SH, Negrin RS, Contag CH. Synergistic antitumor effects of immune cell-viral biotherapy. Science 2006; 311: 1780-1784.

15 Komarova S, Kawakami Y, Stoff-Khalili MA, Curiel DT, Pereboeva L. Mesenchymal progenitor cells as cellular vehicles for delivery of oncolytic adenoviruses. Mol Cancer Ther 2006; 5: 755-766.

16 Hakkarainen T, Sarkioja M, Lehenkari P, Miettinen S, Ylikomi T, Suuronen $\mathrm{R}$ et al. Human mesenchymal stem cells lack tumor tropism but enhance the antitumor activity of oncolytic adenoviruses in orthotopic lung and breast tumors. Hum Gene Ther 2007; 18: 627-641.

17 Raykov Z, Balboni G, Aprahamian M, Rommelaere J. Carrier cell-mediated delivery of oncolytic parvoviruses for targeting metastases. Int J Cancer 2004; 109: 742-749.

18 Garcia-Castro J, Martinez-Palacio J, Lillo R, Garcia-Sanchez F, Alemany R, Madero L et al. Tumor cells as cellular vehicles to deliver gene therapies to metastatic tumors. Cancer Gene Ther 2005; 12: 341-349.

19 Power AT, Bell JC. Cell-based delivery of oncolytic viruses: a new strategic alliance for a biological strike against cancer. Mol Ther 2007; 15: 660-665.

20 Bergman I, Whitaker-Dowling P, Gao Y, Griffin JA. Preferential targeting of vesicular stomatitis virus to breast cancer cells. Virology 2004; 330: 24-33.

21 Hallak LK, Merchan JR, Storgard CM, Loftus JC, Russell SJ. Targeted measles virus vector displaying echistatin infects endothelial cells via alpha(v)beta3 and leads to tumor regression. Cancer Res 2005; 65: 5292-5300.

22 Morizono K, Xie Y, Ringpis GE, Johnson M, Nassanian H, Lee B et al. Lentiviral vector retargeting to P-glycoprotein on metastatic melanoma through intravenous injection. Nat Med 2005; 11: 346-352.

23 Nakamura T, Peng KW, Harvey M, Greiner S, Lorimer IA, James $\mathrm{CD}$ et al. Rescue and propagation of fully retargeted oncolytic measles viruses. Nat Biotechnol 2005; 23: 209-214.

24 Bucheit AD, Kumar S, Grote DM, Lin Y, von MV, Cattaneo RB et al. An oncolytic measles virus engineered to enter cells through the CD20 antigen. Mol Ther 2003; 7: 62-72. 
25 Hammond AL, Plemper RK, Zhang J, Schneider U, Russell SJ, Cattaneo R. Single-chain antibody displayed on a recombinant measles virus confers entry through the tumor-associated carcinoembryonic antigen. J Virol 2001; 75: 2087-2096.

26 Peng KW, Donovan KA, Schneider U, Cattaneo R, Lust JA, Russell SJ. Oncolytic measles viruses displaying a single-chain antibody against CD38, a myeloma cell marker. Blood 2003; 101: 2557-2562.
27 Schuurman HJ, Pierson III RN. Progress towards clinical xenotransplantation. Front Biosci 2008; 13: 204-220.

28 Huppert J, Gresland L, Lazar P. Heterogeneity of chick embryo cells with regard to Newcastle disease virus multiplication. J Gen Virol 1974; 23: 281-287.

29 Cole C, Qiao J, Kottke T, Diaz RM, Ahmed A, Sanchez-Perez L et al. Tumor-targeted, systemic delivery of therapeutic viral vectors using hitchhiking on antigen-specific T cells. Nat Med 2005; 11: 1073-1081. 